\title{
PROPOSTA DE MODELAGEM SIMPLIFICADA PARA VAPOR CHAMBER
}

\author{
Natallie Zilio de SouZa ${ }^{1}$, João Batista Campos Silva ${ }^{1}$, Amarildo TABOne Paschoalini ${ }^{1}$, Márcio AN- \\ TONIO BAZANI ${ }^{1}$.
}

\author{
1. Laboratório de Simulação Numérica, Departamento de Engenharia Mecânica, Universidade Estadual \\ Paulista \\ Avenida Brasil 56, Ilha Solteira SP, CEP: 15385-000 \\ E-mails: natalliezilio@gmail.com,jbcampos@dem.feis.unesp.br, tabone@dem.feis.unesp.br, baza- \\ ni@dem.feis.unesp.br
}

\begin{abstract}
Resumo - Este trabalho apresenta uma proposta de modelagem para um trocador de calor que opera através da ebulição de um fluido de trabalho, objetivando analisar a distribuição de temperatura quando utilizado em conjunto nos sistemas eletrônicos, visto que os componentes eletrônicos estão cada vez menores e dissipando muito mais calor. O trocador de calor estudado é conhecido como vapor chamber. Uma análise numérica utilizando um modelo simplificado de resistências térmicas será realizada. Este modelo será criado para ser utilizado no software ANSYS ${ }^{\circledR}$. A vapor chamber será representada por vários blocos, cada um com uma resistência térmica efetiva, e a contribuição de cada bloco na transferência de calor total será investigada. Uma vez que o desempenho térmico global depende da condutividade térmica de cada bloco, a avaliação poderá dizer quais blocos contribuem para uma melhor eficiência na troca de calor. Os experimentos fornecerão os valores das condutividades térmicas necessários para serem inseridos no modelo computacional, e desta forma, o perfil de temperatura poderá ser encontrado.Este modelo é adequado para a monitorização de problemas que envolvem falhas em componentes eletrônicos devido à alta temperatura.
\end{abstract}

Palavras-chave— ebulição nucleada, transferência de calor, simulação, modelagem, componentes eletrônicos.

\section{Introdução}

Com a evolução dos componentes eletrônicos, estes se tornaram cada vez menores. Isto levou a uma compactação de componentes em placas de circuito impresso, gerando problemas em manter os sistemas estáveis. Com a compactação, os atuais equipamentos eletrônicos dissipam muito mais claor que os antigos. Cada vez mais os aparelhos eletronicos estão menores e realizando mais funções. Deste modo o estudo da transferência térmica do componente é importante para garantir o correto funcionamento destes dispositivos, pois muitas falhas eletrônicas estão relacionadas aos excessivos níveis de temperatura e a fadiga térmica.

Para uma completa adequação destes sistemas é necessário que estudos no campo da transferência de calor e massa, termodinâmica e mecânica dos fluidos sejam realizados. Princípios como a conservação da massa, do movimento linear e angular, do aumento da entropia e da conservação da energia devem ser cuidadosamente analisados e respeitados.

Um projeto térmico de um equipamento eletrônico é uma das partes mais importantes do produto, na qual é efetivamente decisivo para a confiabilidade do mesmo. A verificação de projetos térmicos consiste em ensaios experimentais e simulações computacionais que irão garantir que os resultados satisfaçam ou não os requisitos das especificações do produto.

Um fator bastante importante que deve ser considerado para o controle térmico é o ambiente térmico, que se refere a todas as condições, configurações e fenômenos externos que de algum modo influenciam o projeto térmico de um equipamento eletrônico. Sabe-se que a temperatura é o principal fator responsável por falhas em equipamentos eletrônicos. Ela pode alterar parâmetros como: resistência, indutância, capacitância, constante dielétrica, provocar deterioração do encapsulamento e induzir reações químicas parasitas. Alguns outros fatores que podem gerar falhas são: vibração, poeira, umidade, salinidade, altitude, choque, entre outros.

A ebulição nucleada é um dos mecanismos mais eficientes de remoção de calor de uma superfície aquecida. Sua característica mais importante é a elevada taxa de remoção de calor de superfícies aquecidas com diferencial relativamente reduzido de temperaturas. Em outros termos, a ebulição nucleada se caracteriza por elevados coeficientes de transferência de calor.

Uma das aplicações que mais tem recebido atenção atualmente é o resfriamento de circuitos integrados com alta concentração de potência, nos quais o único meio de adequado resfriamento é a ebulição de um fluido eletricamente neutro, como os componentes halogenados utilizados em aplicações frigoríficas. A mudança de fase com formação de bolhas ocorre em aplicações envolvendo o escoamento do líquido em canais ou exteriormente a superfícies, como na ebulição em película.

A simulação computacional se tornou imprescindível devido à grande quantidade de elementos a serem analisados, inviabilizando um tratamento puramente analítico. Através dela pode-se predizer o desempenho de equipamentos e processos antes mesmo de serem produzidos. 


\section{Objetivos}

Este trabalho pretende definir um modelo numérico capaz de avaliar o desempenho térmico de um trocador de calor que opera através da ebulição de um fluido de trabalho, conhecido como vapor chamber. O intuito do trabalho é estudar o trocador de calor e encontrar a distribuição de temperatura quando ele for utilizado em conjunto nos sistemas eletrônicos. Para isso, o modelo será criado para ser utilizado no programa de simulação numérica computacional, o ANSYS ${ }^{\circledR}$, sendo que ele possibilitará a análise numérica do fluxo de calor através do trocador de calor, bem como uma análise das variáveis de interesse do problema estudado. Uma análise numérica utilizando um modelo simplificado de resistências térmicas será realizada. O trocador de calor será representado por vários blocos, cada um com uma resistência térmica efetiva, e a contribuição de cada bloco na transferência de calor total será investigada.

Pretende-se analisar um protótipo do trocador estudado, para que sejam realizadas as verificações experimentais necessárias para atender as especificações de resfriamento e distribuição de temperatura nos sistemas eletrônicos.A partir do embasamento fornecido pelos experimentos, pretende-se realizar as simulações numéricas, comparando-se os resultados.

\section{Metodologia}

A Figura 1 (Wei, 2006) mostra uma vapor chamber sendo utilizada entre uma fonte de calor (GPU) e um dissipador de calor com aletas (heatsink).

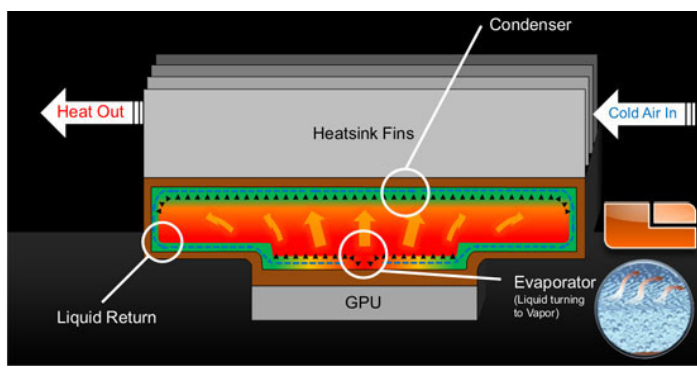

Figura 1. Uso da vapor chamber como trocador de calor

O calor é transmitido efetivamente da fonte de calor para a vapor chamber utilizando o calor latente de evaporação e condensação de um fluido de trabalho. O fenômeno de mudança de fase implica em uma condutividade térmica efetiva elevada da vaporchamber. A Figura 2 (Wei, 2006) mostra as regiões de uma vaporchamber. O calor gerado pela fonte de calor chega até o evaporador que existe em sua parte inferior. O líquido saturado que existe na região denominada wick evapora e transporta o calor adquirido da fonte quente. Este vapor circula na região branca mostrada na figura, e atinge a região do condensador, região esta que fica próxima ao dissipador de calor. No condensador, o calor é rejeitado para o ambiente, fazendo o vapor tornar-se liquido novamente. Este líquido, através do efeito de capilaridade, retorna para o evaporador localizado na fonte de calor, onde se inicia um novo ciclo.

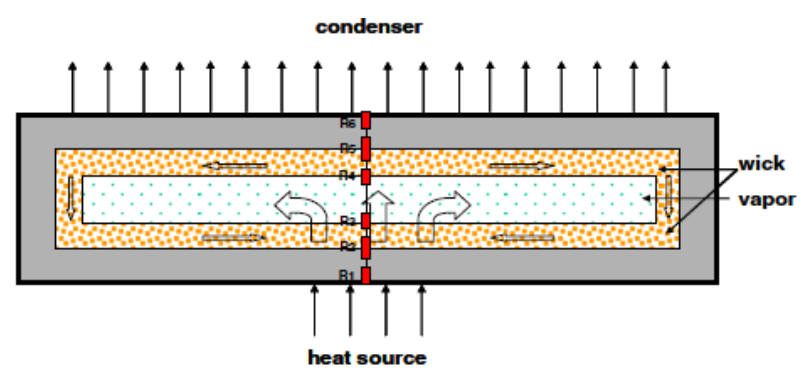

Figura 2. Esquema de funcionamento da vapor chamber

\section{Modelagem Matemática}

A proposta deste trabalho é definir um modelo numérico capaz de avaliar o desempenho térmico de uma vaporchamber. Para isso, a aproximação simplificada de resistências térmicas será utilizada para encontrar o perfil de temperatura. Este modelo será criado para ser utilizado no software $A N S Y S{ }^{\circledR}$ (2008).

No modelo proposto, a vapor chamberserá representada por vários blocos, cada qual com uma resistência térmica efetiva, e a contribuição de cada bloco na transferência de calor total é investigada. Pelo fato de cada região apresentar uma condutividade térmica, pretende-se inserir os valores dessas condutividades de acordo com os dados oriundos de ensaios experimentais. Desta forma, o perfil de temperatura poderá ser encontrado.

Segundo Koito (2008) o domínio computacional é composto por três regiões: a região de vapor, a região denominada wick, que é formada pela combinação de um sólido com um líquido, e a região de parede sólida. Essas regiões compõem a estrutura da vapor chamber, e elas são colocadas entre uma pequena fonte de calor e um grande dissipador. A parte inferior da vapor chamber recebe o calor da fonte, enquanto a parte superior é inteiramente resfriada pelo dissipador de calor.
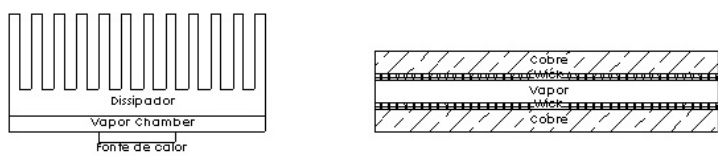

Figura 3. Domínio computacional

A análise de uma vapor chamber se torna complicada devido ao fato de existir mudança de fase de um fluido em seu interior. Devido a isso, existem muitas variáveis envolvidas no problema. $\mathrm{O}$ fato de existir a estrutura denominada wick, que compreende tanto uma fase sólida como uma fase líquida, dificul- 
ta ainda mais a análise. Desta forma, existem diversos mecanismos envolvidos. A troca de calor por condução nas fases sólidas, a troca de calor por mudança de fase na estrutura wick e a convecção que existe devido à movimentação do fluido tornam todo o problema muito complexo (Incropera, 2007).

A proposta é criar um modelo simplificado de resistências térmicas no programa ANSYS ${ }^{\circledR}$ (2008). O intuito deste modelo é predizer a eficiência de uma vapor chamber de forma simples nos problemas que envolvem grandes taxas de fluxo de calor.

Cada região da vaporchamber apresentará uma condutividade térmica, e, sendo assim, pretende-se inserir os valores dessas condutividades de acordo com os dados oriundos de ensaios experimentais.O problema será simplificado, sendo modelado de tal forma que só existe troca de calor por condução.

As condutividades de cada região levam em conta a real troca de calor envolvida. Na região onde existe o vapor, a condutividade deve apresentar um valor elevado na direção do espalhamento lateral, pois deve ser levado em consideração que a troca de calor nessa região é alta, devido ao fenômeno da convecção e mudança de fase.

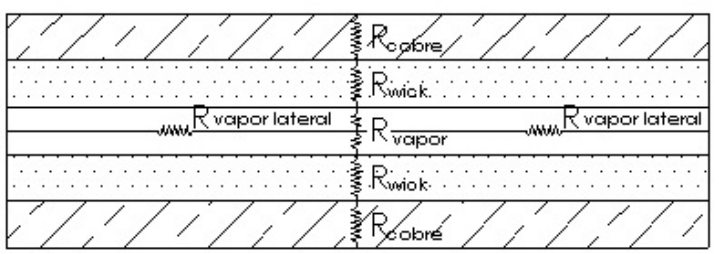

Figura 4. Resistências envolvidas no modelo simplificado

A inserção das condutividades térmicas do cobre, do wick e do vapor no modelo sugerido torna possível avaliar a resistência térmica à propagação de calor de cada região. A resistência térmica de condução por ser encontrada pela seguinte equação:

$$
R_{\text {condução }}=\frac{\Delta T}{q}=\frac{L}{k A}
$$

Para a realização das simulações será utilizado o software ANSYS - ICEPAK, pacote computacional que utiliza o método de volume de controle para a análise de transferência de calor. Para a modelagem em si, foi considerada a figura 4, no qual o domínio computacional foi definido através das resistências térmicas mencionadas e as condições de contorno são as seguintes:Parede lateral direita adiabática; Parede lateral esquerda adiabática; Superfície inferior com fluxo de calor uniforme; Superfície superior com sistema de aletas sendo resfriada através da convecção forçada pelo túnel de vento.

O coeficiente de troca de calor convectivo entre o sistema aletado e a corrente de ar dentro do túnel de vento será calculado através da simulação de um escoamento interno pelo ANSYS - ICEPAK.
As simulações do túnel de vento levarão em conta: as equações da continuidade, as equações da conservação da quantidade de movimento, equação de energia e modelos de turbulência fornecidos pelo software (k-epsilon, RNG, zero equation). A validação desses resultados numéricos será feita através de ensaios experimentais no aparato mostrado na figura 7.

Uma análise criteriosa sobre a malha de volume de controle será feita através da convergência dos resultados que serão comparados com os resultados experimentais.

\section{Proposta Experimental}

A proposta do experimento é avaliar um protótipo de uma vaporchamber para que sejam realizadas as verificações experimentais necessárias para atender as especificações de resfriamento e distribuição de temperatura nos sistemas eletrônicos. O experimento consiste em utilizar a vaporchamber em um conjunto de fonte de calor e dissipador em situação de potência e escoamento externo controlados. Uma vez observado o perfil de temperatura ao longo das faces inferior e superior do aparato, as resistências térmicas poderão ser calculadas. A vapor chamber que será utilizada nos ensaios experimentais está apresentada na Figura 5. O fluido de trabalho é a água, e o material é o cobre.

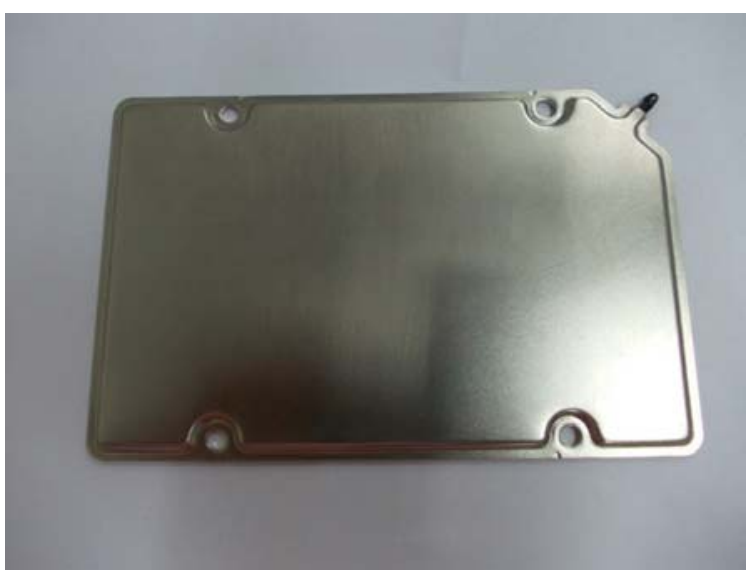

Figura 5. Vapor chamber

O conjunto contendo a vapor chamber, fonte de calor e dissipador estará disposto no interior de um túnel de vento, e a fim de garantir uma condição adiabática entre a face inferior, em torno da fonte de calor e o meio, será instalada uma câmara com vácuo. Para monitorar a temperatura nesta face, a mesma será dividida simetricamente em quatro quadrantes e 12 termopares serão instalados em um quadrante. O posicionamento dos termopares pode ser observado na Figura 8. 


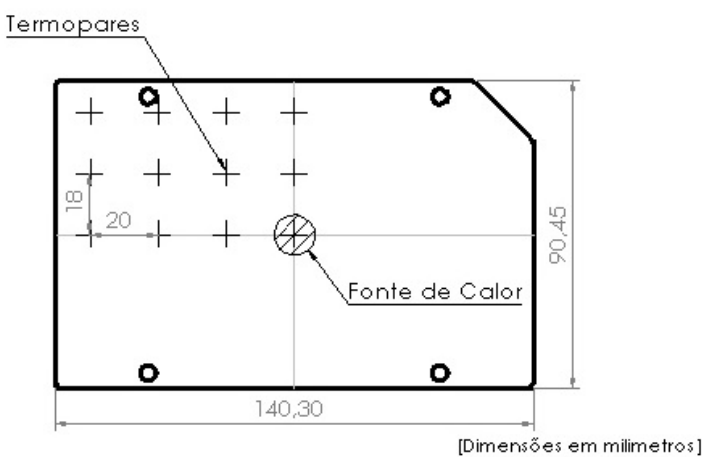

Figura 6. Posicionamento dos termopares

A potência presente na fonte de calor poderá ser configurada, e a face superior será monitorada por um termovisor através de imagens térmicas.

O túnel de vento possui a seção de testes com área de 360 x $60 \mathrm{~mm}^{2}$, o escoamento é criado através de sucção por um exaustor, podendo ter velocidade entre 0 e $4 \mathrm{~m} / \mathrm{s}$, que são velocidades geradas por ventiladores em componentes eletrônicos utilizados em sistemas de telecomunicações. O número de Reynolds será baseado no diâmetro hidráulico do túnel de vento. Como foi dito anteriormente, a transferência de calor através da convecção forçada em sistemas de telecomunicações já chegou no seu limite, por isso um túnel de vento de baixa turbulência é suficiente, pois a maior parte da transferência de calor será feita pela vapor chamber através do calor latente gerado pela mudança de fase do fluido de trabalho. Um esquema simplificado do experimento é apresentado na Figura 7.

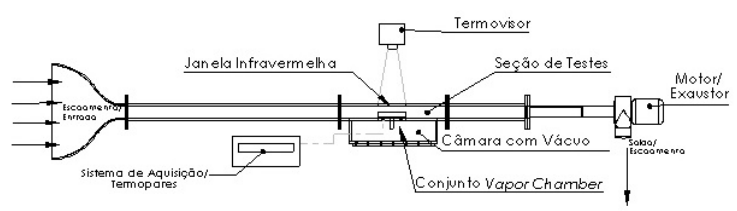

Figura 7. Túnel de vento

Com essa metodologia espera-se através dos resultados obtidos subsidiar o cálculo das resistências térmicas e validar o modelo numérico proposto.

\section{Conclusão}

O estudo de novas formas de resfriamento em componentes eletrônicos se torna importante devido ao fato dos componentes estarem cada vez menores e realizando muitas funções. Com o aumento na dissipação de energia, os riscos de falhas aumentam e é nesse contexto que surge o interesse de analisar tanto numericamente quanto experimentalmente os trocadores de calor eficientes na remoção de altos fluxos de calor.

O presente trabalho propõe investigar um trocador de calor conhecido como vapor chamber, que opera através da ebulição de um fluido de trabalho. O fato de existir o fenômeno de mudança de fase implica numa troca de calor muito maior do que a que ocorreria somente com convecção forçada, pois os efeitos do calor latente são muito significativos.

A partir dos conhecimentos adquiridos com o estudo da teoria envolvida, juntamente com o modelo matemático proposto (modelo simplificado de resistências térmicas) e através dos experimentos sugeridos, será possível chegar numa investigação satisfatória da eficiência térmica de uma vaporchamber

\section{Agradecimentos}

Agradecimentos especiais a FAPESP pela viabilização da apresentação desse trabalho.

\section{Referências Bibliográficas}

ANSYS, Inc. ANSYS alrights reserved, 2008.

CAREY, V. P. (1992) Liquid-Vapor PhaseChangePhenomena. Taylor \& Francis, USA,.

CHI S. (1976) Heat Pipe Theory and Practice, Hemisphere Publishing Corporation.

COLLIER, J. G., J. R. Thome (1996) Convective Boling and Condensation, $3^{\text {rd }}$ ed., Oxford University Press, New York,.

DUNN P.D., REAY D.A. (1994)Heat Pipe, 4th edition, Pergamon Press,.

FAGHRI A. (1995) Heat Pipe Science and Technology, Washington, DC: Taylor \& Francis, ch. 4.

FOX, R. W., A. T. MCDONALD, P. J. (2003) Pritchard. Introduction to Fluid Mechanics, $6^{\text {th }}$ ed. Wiley, New York.

GRUBB K., “CFD Modeling of a Therma-Base Heat Sink”, 8th International FLOTHERM User Conference, Las Vegas, USA, 13-14, May 1999.

HWANG G. S., NAM Y., FLEMING E., DUSSINGER P., JU Y. S., KAVIANY M. 2010, Int. J. Heat Mass Transfer, 53 2662-2669.

DOI: 10.1016/j.ijheatmasstransfer.2010.02.046

INCROPERA, Frank P. (2007) Introduction to Heat Transfer, $6^{\text {th }}$ ed. New York: John Wiley \& Sons,.

KOITO Y., IMURA H., MOCHIZUKI M., SAITO Y., TORII S., 2006 Appl. Thermal Eng., 26, 1669-1676. DOI:

10.1016/.applthermaleng.2005.11.012

KOITO Y., IMURA H., MOCHIZUKI M., SAITO Y., TORII S., 2006, JSME Int. J., Ser. B, 49, 1233-1240.

MAXWELL J.C., A Treatise on Electricity and Magnetism, (1954) Vol. 1, 3rd edition, reprinted by Dover, New York.

MOCHIZUKI M., SAITO Y., KIYOOKA F., NGUYEN TH., WU X. P., NGUYEN TI., WUTTIJUMNONG V. 2007, Proc. 14th Int. Heat Pipe Conference, , pp. 221-226.

MOCHIZUKI M., MASHIKO K., SAITO Y., NGUYEN TH., WU X. P., NGUYEN TI., WUTTIJUMNONG V., 2008, Proc. 9th Int. Heat Pipe Symposium, , pp. 39-48. 
NUKIYAMA, S., J. 1966, Japan Soc. Mech. Eng., 37, 367, 1934 Translation: Int. J. Heat Mass Transfer, 9, 1419. DOI:

10.1016/0017-9310(66)90138-4

PRASHER R., (2003) “A Simplified Conduction Based Modeling Scheme for Design Sensitivity Study of Thermal Solution Utilizing Heat Pipe and Vapor Chamber Technology”, J. Electronic Packaging, Vol. 125, pp. 378-385,. DOI: $10.1115 / 1.1602479$

ROHSENOW, W. M., Trans. ASME, 74, 969, 1952.

SAUCIUC I., CHRYSLER G., MAHAJAN R., PRASHER R., (2002) "Spreading in the Heat Sink Base: Phase Change Systems or Solid Metals??," IEEE Transactions on Components and Packaging technologies, Vol. 25, pp. 621-628,.

TSAI T. E., WU H. H., CHANG C. C., CHEN S. L., 2010, Int. Commun. Heat Mass Transfer, 37, 484-489. DOI:

10.1016/j.icheatmasstransfer.2010.01.002

VADAKKAN U., CHRYSLER G., SANE S., 2005, "Silicon/Water Vapor Chamber as Heat Spreaders for Microelectronic Packages”, IEEE SEMITHERM Symposium, pp. 182-186.

VADAKKAN U., MURTHY J., GARIMELLA S., 2003, "Transient Analysis of Flat Heat Pipes", ASME Summer Heat Transfer Conference, July 21-23, , Las Vegas, Nevada, USA.

WANG J. C., WANG R. T., CHANG T. L., HWANG D. S., 2010 Int. J Heat Mass Transfer, 53, 3990-4001. DOI:

10.1016/j.ijheatmasstransfer.2010.04.001

WEI J., CHA A., COPELAND D., (2003) "Measurement of vapor Chamber Performance", IEEE SEMI-THERM Symposium, pp.191-194.

WONG S. C., HSIEH K. C., WU J. D., HAN W. L., (2010) Int. J. Heat Mass Transfer, 53, 23772384. DOI:

10.1016/j.ijheatmasstransfer.2010.02.001

YAMAGATA, K., F. KIRANO, K. NISHIWAKA, H. MATSUOKA, (1955),Mem. Fac. Eng. Kyushu, 15, 98, 